\title{
Integrity of Nonviral Fragments in Recombinant Tomato bushy stunt virus and Defective Interfering RNA Is Influenced by Silencing and the Type of Inserts
}

\author{
Xueyan Zhong, Hesheng Hou, and Wenping Qiu \\ Southwest Missouri State University at Mountain Grove, Department of Fruit Science, 9740 Red Spring Road, \\ Mountain Grove 65711, U.S.A.
}

Submitted 20 September 2005. Accepted 30 March 2005.

\begin{abstract}
Recombinant plant viruses have the propensity to remove foreign inserts during replication. This process is virusspecific and occurs in a host-dependent manner. In the present study, we investigated the integrity of foreign inserts in recombinant plant viruses using a model system consisting of Tomato bushy stunt virus (TBSV) and its defective interfering RNA (DI). These were tested in Nicotiana benthamiana plants that were either wild type or transgenic for the green fluorescent protein (GFP) gene. GFP-derived inserts were retained in the recombinant TBSV and DI population that were inoculated onto GFPtransgenic $N$. benthamiana plants in which silencing of the GFP transgene was initiated, but they were removed from the virus and DIs that were maintained on wild-type plants. A foreign insert derived from an endogenous $N$. benthamiana gene encoding the $H$ subunit of the magnesium chelatase $(\mathrm{NbChlH})$ was deleted, whereas the fragment of an RNA-dependent RNA polymerase gene (NbRdRP1m) was retained in the recombinant TBSV population. These results demonstrate that the recombination of TBSV to remove nonviral fragments is influenced by silencing and the type of inserts.
\end{abstract}

Virus-induced gene silencing (VIGS) is an RNA-specific antiviral defense response that interferes with the infection process of an invading virus in plants (Ratcliff et al. 1997; Waterhouse et al. 2001). VIGS leads to the degradation of cognate mRNA of a gene if a fragment of the same gene is embedded in the genome of a plant virus. On the basis of this innate plant defense phenomenon, a number of plant viruses have been modified and employed as gene delivery vehicles to silence host genes (Faivre-Rampant et al. 2004; Hiriart et al. 2002; Liu et al. 2002). The putative function of a host gene then can be inferred by the new phenotype (Baulcombe 1999). For instance, the phytoene desaturase (PDS) gene was systemically

Corresponding author: W. Qiu; Telephone: E-mail: weq070f@smsu.edu; +1.417.926.4105; Fax: +1.417.926.6646.

Current address of X. Zhong: City of Hope National Medical Center Beckman Research Institute Division of Biology, Lippman B134 Duarte, CA 91010 , U.S.A.

Current address of H. Hou: Department of Biology, Liaoning Normal University, Dalian, Liaoning 116029, China. silenced in leaves and tubers of potato that were infected with Potato virus $X$ (PVX) containing a fragment of the PDS gene, demonstrating that PVX can be used as a functional genomics tool for studying the function of host genes (Faivre-Rampant et al. 2004). Other plant viruses, such as Tobacco mosaic virus (TMV) and Tobacco rattle virus (TRV), also have been used as viral gene vectors to reduce the expression level of endogenous host genes to elucidate their functions (Hiriart et al. 2002; Liu et al. 2002).

In tissues undergoing VIGS, it is reasonable to presume that both viral RNAs and nonviral inserts are subjected to elimination by the VIGS pathway. For example, neither the green fluorescent protein (GFP) gene inserted into the PVX viral genome nor genomic sequences of PVX were detected in tissues where silencing of the transgenic GFP gene was fully activated by VIGS (Ruiz et al. 1998; Thomas et al. 2001). In other studies, however, the mRNAs of target genes were degraded, whereas the chimeric viruses containing the fragments of the same genes were not completely eliminated by the VIGS machinery (Hiriart et al. 2003; Qiu et al. 2002; Ratcliff et al. 2001). It is noted that, in these studies, the presence of chimeric viruses was examined only with virus gene-specific probes in the RNA gel blot assays (Hiriart et al. 2003; Ratcliff et al. 2001); therefore, the maintenance of the foreign inserts in these plant virus gene vectors is unclear.

It is known that recombinant viruses frequently delete foreign inserts from their genomes, which may be an innate property of plant viruses for maintaining their genome integrity (Pogue et al. 2002). The propensity of recombinant plant viruses to eliminate nonviral fragments was illustrated clearly in experiments in which virus populations were transferred from plants to plants through sequential passages (Pogue et al. 2002; Rabindran and Dawson 2001; Scholthof et al. 1996). Therefore, it is an interesting question whether VIGS affects recombination to remove foreign inserts when a plant virus is used as a gene delivery vector to silence a transgene or a host gene. The answer to this question may reveal possible interconnections between VIGS and viral recombination pathways.

Tomato bushy stunt virus (TBSV) is the type member of the Tombusvirus genus in the family Tombusviridae (Russo et al. 1994). It encodes five major proteins: P33, P92, P41, P22, and P19. P33 and P92 are two key components of the viral replication complex (Scholthof et al. 1995b). The P41 capsid protein $(\mathrm{CP})$ is dispensable for virus replication (Scholthof et 
al. 1993), but assists long-distance vascular spread of the virus in Nicotiana benthamiana plants (Desvoyes and Scholthof 2002; Qu and Morris 2002). P22 represents the typical cellto-cell movement protein, whereas P19 has host-specific effect on virus spread. The presence of P19 causes a lethal systemic necrosis in $N$. benthamiana plants, but its absence does not affect replication and initial systemic infection (Scholthof et al. 1995a and c). P19 was shown to be a suppressor of post-transcriptional gene silencing in N. benthamiana plants (Qiu et al. 2002; Qu and Morris 2002; Silhavy et al. 2002; Voinnet et al. 1999); however, it only delays the onset of VIGS and is unable to suppress defective interfering RNA (DI)-mediated gene silencing (Qiu et al. 2002). TBSV has been widely used as a plant viral gene vector to express genes of interest inserted in place of the CP gene (Hou and Qiu 2003; Qiu et al. 2002; Scholthof 1999; Sit et al. 1998). However, TBSV inclines to remove foreign inserts frequently (Scholthof 1999) and also recombines efficiently with functional CP mRNAs that accumulated in transgenic plants to regenerate wild-type TBSV (Borja et al. 1999).

An additional intriguing feature of TBSV is its propensity to generate DIs during the infection process (Hillman et al. 1987; White and Morris 1999). DIs are derived from TBSV but do not contain coding regions for expressing functional proteins. As a result, DIs rely on the helper TBSV for replication and movement. Numerous studies suggest that de novo generation of DIs most likely occurs through a series of replicase-mediated deletion events which result in multiple stepwise deletions of various segments of the viral genome (Szittya et al. 2002; White and Morris 1999). Moreover, previous results demonstrated that larger DIs with potential VIGS target sequences are prone to degradation by the VIGS machinery and, consequently, are eliminated from the DI population, whereas shorter DIs are weak targets of VIGS pathways and are retained in the population (Szittya et al. 2002). These results suggest that VIGS shapes the composition of DI populations by eliminating DIs that contain sequences that are vulnerable targets of the VIGS pathway (Szittya et al. 2002). Interestingly, results from another study showed that a chimeric DI-P, which contains the $3^{\prime}$ end portion (P) of the GFP gene, was able to silence GFP transgene efficiently and, at the same time, also accumulated abundantly in tissues undergoing silencing of the target GFP transgene (Hou and Qiu 2003; Qiu et al. 2002). This apparent discrepancy suggests that the influence of VIGS on DI formation and evolution may be more complex than originally presumed.

In this study, we used recombinant TBSV and DIs that contained a fragment of the GFP gene and GFP-transgenic $N$. benthamiana plants as a model to investigate the potential influence of gene silencing on the integrity of nonviral fragments inserted in the genome of recombinant TBSV and DIs. The results demonstrate that GFP sequences were removed from chimeric TBSV and DIs only in wild-type plants, but were retained in the genome of recombinant TBSV and DIs in transgenic plants in which silencing of the GFP transgene was initiated. We also investigated the integrity of nonviral fragments that were derived from native host genes in recombinant TBSV in wild-type $N$. benthamiana plants. A foreign insert from an endogenous $\mathrm{NbChlH}$ gene encoding the $\mathrm{H}$ subunit of the magnesium chelatase was removed from the chimeric TBSV virus, whereas a fragment of the RNAdependent RNA polymerase (NbRdRPlm) gene was retained in recombinant TBSV virus in the upper, systemically infected leaves in $N$. benthamiana plants. These results suggest that the gene-silencing pathway and the type of host gene influence the removal or retention of nonviral fragments inserted in recombinant TBSV and DIs.

\section{RESULTS}

\section{The integrity of GFP-derived foreign inserts in a recombinant TBSV virus.}

In a previous study (Qiu et al. 2002), a recombinant TBSV virus pTG-p19/wt was constructed that contained a foreign insert derived from the original GFP gene of Aequorea victoria (GenBank accession no. L29345) (Fig. 1A). The pTG-p19/wt virus is able to induce silencing of the GFP gene in transgenic $N$. benthamiana plants transformed with the identical GFP gene, mostly in newly emerging leaves as a result of incomplete suppression of VIGS by P19 (Qiu et al. 2002). This formed an experimental system to test whether gene silencing will affect the integrity of a foreign insert in the recombinant TBSV. The other recombinant TBSV virus, pTsG-p19/wt, was used as a negative control because the synthetic version of GFP (sGFP) gene in pTsG-p19/wt is an extensively modified version of the original GFP gene and does not share stretches of homologous sequences with the GFP transgene (Chiu et al. 1996). Both GFP and sGFP genes are the same size.

After GFP-transgenic $N$. benthamiana plants were inoculated with pTG-p19/wt transcripts, red fluorescence emerged around the borders of local lesions on the inoculated leaves and eventually expanded to newly emerging leaves under UV light. In contrast, mock-inoculated GFP-transgenic N. benthamiana plants remained green fluorescent under UV light (data not shown). A number of previous results proved that the transition from green to red fluorescence correlated positively with the degradation of GFP mRNAs (Hou and Qiu 2003; Qiu et al. 2002; Ruiz et al. 1998; Thomas et al. 2001). Therefore, the observed fluorescence transition indicated that the pTG-p19/wt virus induced silencing of the GFP transgene, although not completely. The incomplete silencing is due to the presence of P19. In contrast, red fluorescence did not emerge on pTsGp19/wt virus-infected GFP-transgenic $N$. benthamiana plants, confirming that no GFP gene silencing was initiated because sGFP does not share homologous sequences with the GFP transgene.

RNA gel blot analysis showed that, in inoculated leaves, the GFP insert was detected in the pTG-p19/wt virus population and the sGFP insert was detected in the pTsG-p19/wt virus population on both GFP-transgenic and wild-type $N$. benthamiana plants in five experiments (Fig. 1B). In upper, systemically infected leaves, GFP inserts were no longer detectable in the pTG-p19/wt virus population on wild-type plants, but were detected in the pTG-p19/wt virus population on GFP-transgenic plants in three of five experiments (Fig. 1B). In contrast, in upper, systemically infected leaves, sGFP was not detected in the pTsG-p19/wt virus population on either GFP-transgenic or wild-type plants, except for one experiment. Previous results verified that transgenically expressed GFP mRNA was below detectable level in RNA gel blot assays when a standard method was adopted to obtain total RNA from GFP-transgenic $N$. benthamiana plants for detecting abundantly accumulating viral RNAs, as exemplified in a previous study (Hou and Qiu 2003). Therefore, the bands on the left-bottom panel of an RNA gel blot (Fig. 1B) that signify hybridization with the GFP-specific probes were derived from viral RNAs that contained the GFP fragments, not from the endogenous GFP transgene mRNAs (the same is true for Figure $2 \mathrm{~B}$, discussed in the following section).

In summary, sGFP inserts were removed from recombinant pTsG-p19/wt viruses on either wild-type or GFP-transgenic plants, and GFP inserts were deleted from the recombinant pTGp19/wt virus population only on wild-type plants, but were retained in the recombinant pTG-p19/wt viruses on GFP-transgenic plants in which silencing of that GFP gene was initiated. 
The integrity of GFP-derived foreign inserts in recombinant DI-P.

Results from a previous study (Qiu et al. 2002) demonstrated that a chimeric DI-P that contains a fragment ( $3^{\prime}$ proximal region from nucleotide 489 to 716 ) of the GFP gene was able to efficiently induce silencing of the GFP transgene in $N$. benthamiana plants in the presence of TBSV or a TBSVderived construct (pHS157) which is incapable of expressing the p19 protein (Scholthof et al. 1995c). This suggests that the TBSV p19 protein is unable to suppress DI-P-mediated gene silencing (Qiu et al. 2002). It was noted that two distinct bands of DI-P were observed on the RNA gel blot in the presence of TBSV, whereas only one lower band of DI-P was detected in the presence of pHS157 in the upper, systemically infected leaves (Qiu et al. 2002). In this study, we employed these pre- viously constructed recombinant viruses and DI-P to further investigate how gene silencing affects DI-P accumulation (Fig. 2A). Transcripts from these constructs were inoculated onto GFP-transgenic $N$. benthamiana plants on which silencing of the GFP transgene was expected to be initiated, and onto wildtype $N$. benthamiana plants that were used as a control. The virus populations also were passed through GFP-transgenic plants two consecutive times to exert selection pressure on the DI-P population and, in parallel, through wild-type plants as a control.

At 5 days postinoculation (dpi), GFP-transgenic and wildtype plants inoculated with mixed TBSV and DI-P transcripts or with mixed pHS157 and DI-P transcripts began to show systemic symptoms typical of TBSV and DI infection. At $15 \mathrm{dpi}$, red fluorescence emerged prominently along the major veins

A
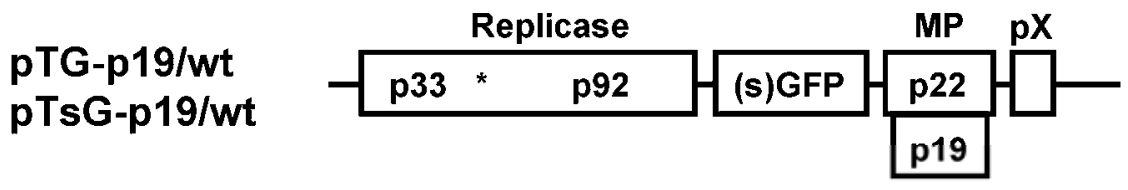

B

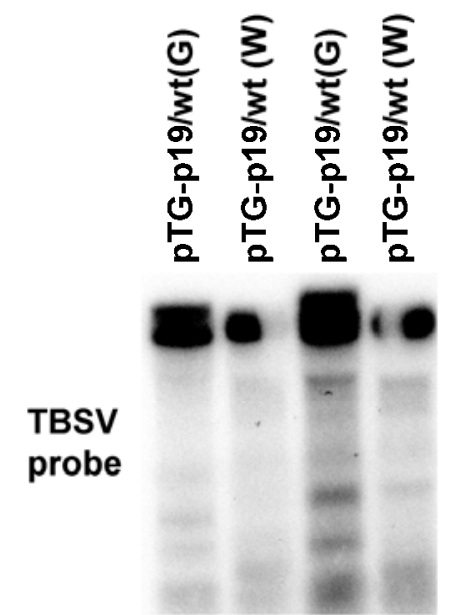

$\begin{array}{llll}5 / 5 & 5 / 5 & 4 / 5 & 4 / 5\end{array}$

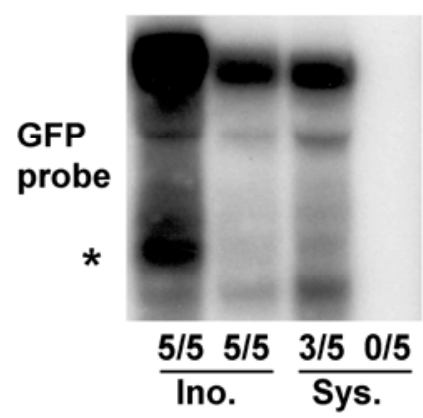

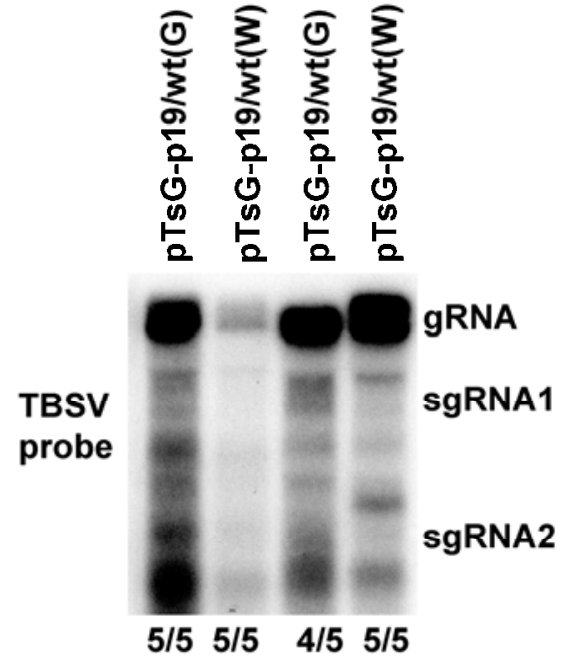

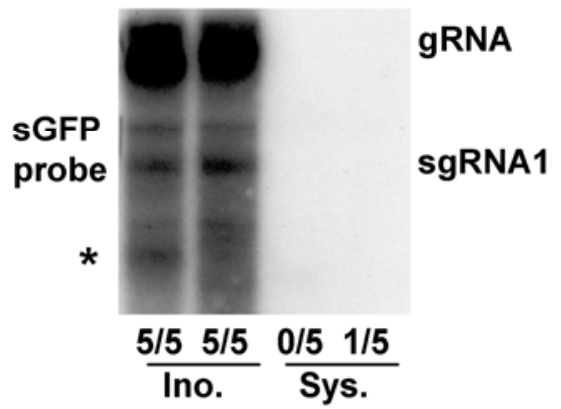

Fig. 1. Integrity of the green fluorescent protein (GFP) gene and a synthetic version of GFP (sGFP) gene in recombinant Tomato bushy stunt virus (TBSV) viruses. A, Schematic illustration of two recombinant TBSV viruses, pTG-p19/wt and pTsG-p19/wt. The GFP and sGFP genes were inserted in place of the capsid protein (CP) gene to form the pTG-p19/wt and pTsG-p19/wt constructs, respectively. Rectangles represent coding regions of TBSV genome. Lines denote nontranslated regions. The function of proteins encoded by TBSV is listed on top of the diagram. MP: movement protein. B, RNA gel blot analysis of total RNA extracted from GFP-transgenic Nicotiana benthamiana plants (G) and wild-type N. benthamiana plants (W) that were inoculated with transcripts of pTG-p19/wt and pTsG-p19/wt. Total RNA was isolated from inoculated leaves (Ino.) at 7 days postinoculation (dpi) and from upper, systemically invaded leaves (Sys.) at 14 dpi. ${ }^{32} \mathrm{P}-\mathrm{dCTP}$-labeled GFP-, $s G F P$-, and TBSV-specific probes are listed on the left side of the blots. The relative positions of TBSV genomic RNA (gRNA), subgenomic RNA 1 (sgRNA1), and subgenomic RNA 2 (sgRNA2) are indicated on the right side of the blots. The RNA blot represents one of five experiments. Times that gene-specific signals were detected out of five experiments are listed at the bottom of the blots. The bands indicated by an asterisk on two bottom blots likely represent defective interfering RNAs. 
of upper leaves in GFP-transgenic $N$. benthamiana plants. Red fluorescence eventually spread through the entire GFP-transgenic plant. The phenotypic exhibition was essentially same as that shown in a previous publication (Qiu et al. 2002). Similarly, DI-P-induced silencing of the GFP gene also was observed on the GFP-transgenic plants that were inoculated with sap inoculum following each sequential passage of the DI-P population, indicating the maintenance of GFP sequence in the DI.

In the RNA gel blot analysis, a GFP-specific probe was used to detect the presence of the GFP fragment in the DI-P population, and a TBSV-specific probe was used to detect the presence of TBSV virus to ensure that the absence of DI-P was not due to the absence of the helper virus. RNA gel blot analyses showed that DI-P accumulated similarly in the presence of P19 (i.e., TBSV) and in the absence of P19 (i.e,. pHS157) in the transcript-inoculated leaves on both GFP-transgenic and wildtype plants (Fig. 2B). These results indicate that DI-P accumulated to a similar level in upper, systemically infected leaves on both GFP-transgenic and wild-type $N$. benthamiana plants.

After the virus population was passed through both host plants for the first time, DI-Ps were detected in the inoculated leaves and in upper, systemically infected leaves of both GFPtransgenic and wild-type plants (Fig. 2B, first transfer). These results are similar to those obtained from transcript-inoculated plants (Fig. 2B, RNA inoculation). The DI-P was not detected in the inoculated leaf of wild-type plants that were infected with pHS157 and DI-P in this RNA gel blot assay, but was detected in two other experiments (data not shown). After the same virus population was passed through both host plants for the second time, DI-Ps were detected in the inoculated and upper, systemically infected leaves only on GFP-transgenic plants, but no longer in the same tissues on wild-type plants (Fig. 2B, second transfer). Additional RNA gel blot assays of total RNA that was extracted from mock-inoculated GFPtransgenic plants by the same method confirmed that the bands detected by the GFP-specific probe were not derived from the endogenous GFP mRNAs (data not shown).

RNA gel blot analysis using a TBSV-specific probe indicated that TBSV genomic RNAs and DIs were present in all tissues tested (Fig. 2B). These results demonstrated that foreign GFP fragments in DI-Ps were not present in the DI population after the population was transferred through wild-type plants two times, but were maintained in the DI population after the population was transferred through GFP-transgenic plants two times. The presence of P19 had no obvious influence on the composition of the DI population. The same results were observed in three independent experiments.

Fig. 2. Integrity of the green fluorescent protein (GFP) gene in the recombinant Tomato bushy stunt virus (TBSV) defective interfering RNA (DI) population. A, Schematic illustration of the genome organization of TBSV, its mutant pHS157, and DI-P. DI-P was generated by inserting the $3^{\prime}$ portion $(\mathrm{P})$ of the GFP gene between region II and III of a prototypic DI that is composed of four regions (I through IV) derived from the TBSV genome. B, RNA gel blot analysis of total RNA that was isolated from inoculated leaves (Ino.) and from upper, systemically infected leaves (Sys.) of GFP-transgenic Nicotiana benthamiana plants (G) and wild-type $N$. benthamiana plants (W) at 7 days postinoculation. The plants were inoculated with transcripts of DI-P and TBSV or DI-P and pHS157 at a ratio of 1:2. The virus population was passed through the same type of plants for two consecutive times (first and second transfer). The ${ }^{32} \mathrm{P}-\mathrm{dCTP}$ labeled GFP- and TBSV-specific probes are listed on the left side of the blots. The relative positions of TBSV genomic RNA (gRNA), subgenomic RNA 1 (sgRNA1), subgenomic RNA 2 (sgRNA2), and DIs are indicated on the right side of the blots.
The integrity of two endogenous host genes in recombinant $\mathrm{TBSV}$ viruses.

Previous studies showed that foreign inserts were frequently deleted from the recombinant TBSV viruses during virus multiplication (Scholthof 1999; Scholthof et al. 1993). In those studies, nonviral fragments were derived from widely used re-

A

TBSV

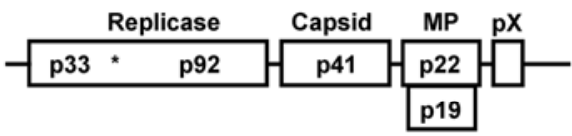

pHS157

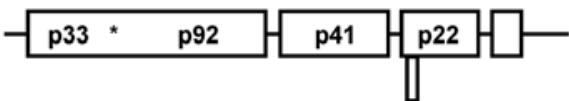

DI-P

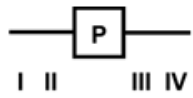

B
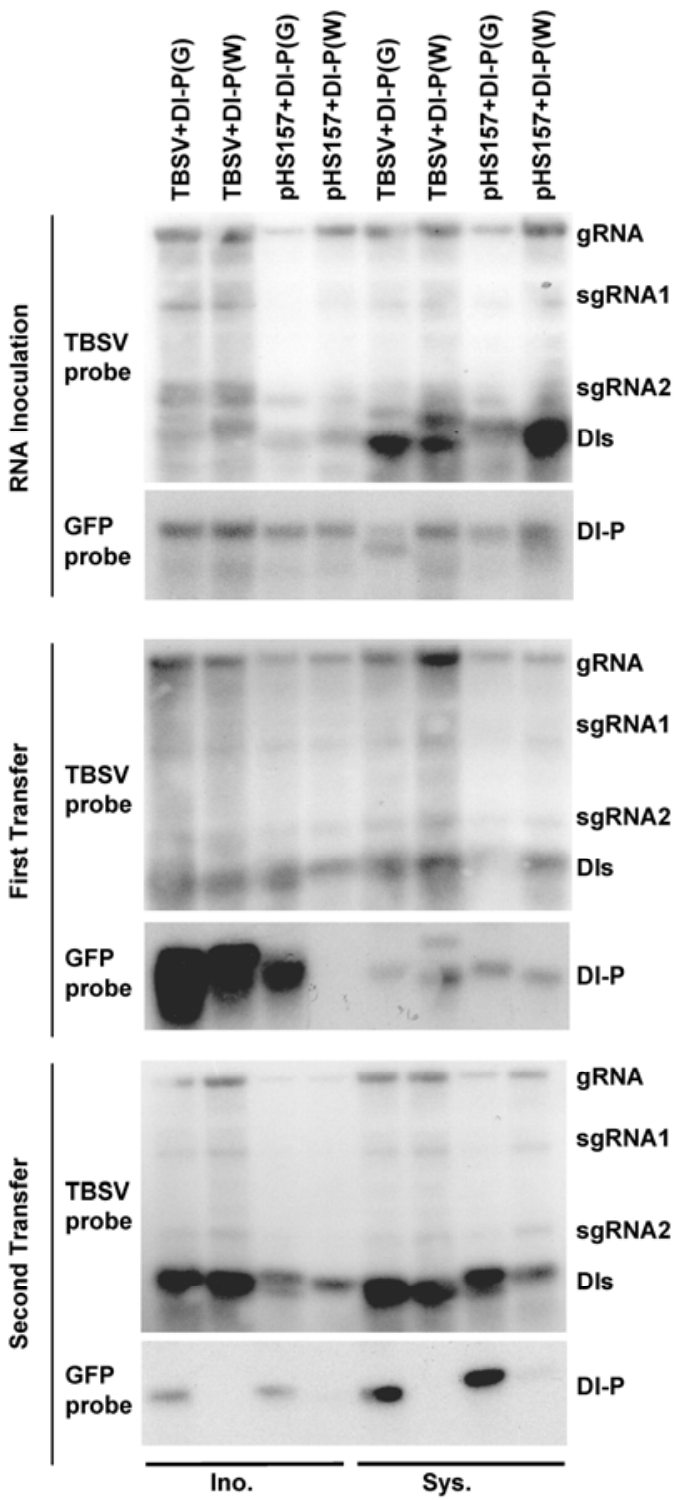

Vol. 18, No. 8, 2005 / 803 
porter genes, such as $\beta$-glucuronidase (GUS) and chloramphenicol acetyltransferase (CAT) genes. Little is known about how the recombination pathway affects the integrity of endogenous host genes as foreign inserts in recombinant TBSV viruses. We selected $\mathrm{NbChlH}$ and NbRdRPIm of $N$. benthamiana plants for this study. The $\mathrm{NbChlH}$ gene encodes the $\mathrm{H}$ subunit of the magnesium chelatase that is a key enzyme for the synthesis of chlorophyll (Walker and Willows 1997). The 776-bp fragment of the NbRdRPIm gene isolated in this study is identical to the corresponding sequence (nucleotide 2,561 to 3,336) of the RdRPIm gene (GenBank accession no. AY5743741) that recently was identified in $N$. benthamiana plant (Yang et al. 2004). The 546-bp fragment of $\mathrm{NbChlH}$ and the 776-bp fragment of NbRdRPIm were inserted in place of the TBSV CP gene to create the recombinant virus pTChlH-p19/wt and pTRdRP1m-p19/wt (Fig. 3A). It previously was shown that $N$. benthamiana plants displayed patchy chlorosis after they were infected with recombinant TMV containing the $\mathrm{NbChlH}$ fragment, indicating that the endogenous $\mathrm{NbChlH}$ gene was silenced upon TMV infection (Hiriart et al. 2002). Throughout three experiments in this study, $N$. benthamiana plants did not show chlorosis on the pTChlH-p19/wt-infected plants. Similarly, no abnormalities other than typical symptoms of TBSV infection were observed on the pTRdRP1m-p19/wt-infected $N$. benthamiana plants.

RNA gel blot assays demonstrated that TBSV viral RNAs were detected in both inoculated and upper, systemically infected leaves (Fig. 3B), suggesting that the TBSV virus was present in the infected tissues. When the same blots were hybridized with the $\mathrm{NbChlH}$-specific probe, the $\mathrm{NbChlH}$ fragment in the pTChlH-p19/wt was detected only in the inoculated leaves, not in the upper, systemically infected leaves (Fig. 3B). The same results were obtained in three independent experiments. RNA gel blots that were hybridized with an

A
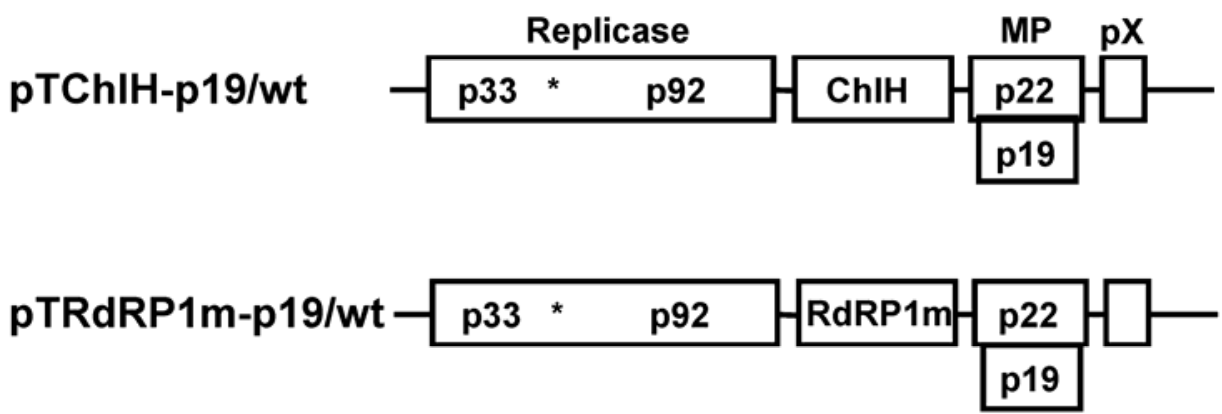

B

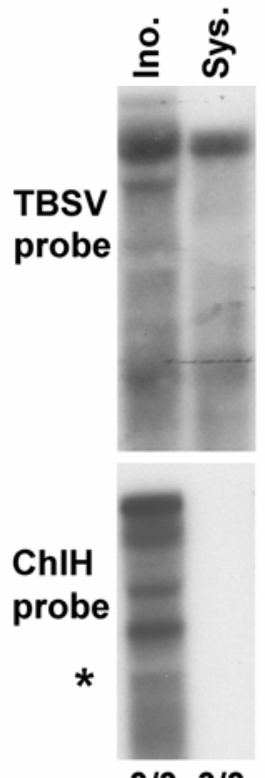

$3 / 3 \quad 3 / 3$

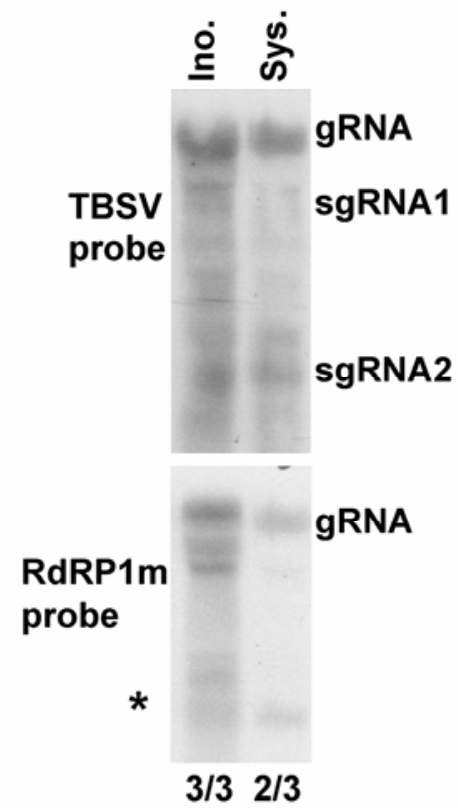

Fig. 3. Integrity of endogenous host gene-derived fragments that were inserted in recombinant Tomato bushy stunt virus (TBSV) viruses, pTChlH-p19/wt and pTRdRP1m-p19/wt, in Nicotiana benthamiana plants. A, Schematic diagram of two recombinant viruses, pTChlH-p19/wt and pTRdRP1m-p19/wt. pTChlH-p19/wt contains a 549-bp fragment of the $\mathrm{NbChlH}$ gene encoding the $\mathrm{H}$ subunit of the magnesium chelatase of $\mathrm{N}$. benthamiana plant in place of the capsid protein (CP) gene. In pTRdRP1m-p19/wt, the TBSV CP gene was replaced by a 776-bp fragment of the NbRdRP1m gene of $N$. benthamiana plant. B, RNA gel blot analysis of total RNA extracted from wild-type $N$. benthamiana plants that were inoculated with pTChlH-p19/wt and pTRdRP1m-p19/wt transcripts. Total RNA was isolated from inoculated leaves (Ino.) at 7 days postinoculation (dpi) and from upper, systemically invaded leaves (Sys.) at 14 dpi.

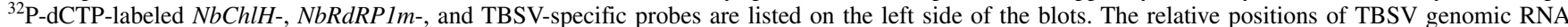
(gRNA), subgenomic RNA 1 (sgRNA1), and subgenomic RNA 2 (sgRNA2) are indicated on the right side of the blots. The RNA gel blot represents one of three repeated experiments. Times that gene-specific signals were detected out of three experiments are listed at the bottom of the blots. The bands indicated by an asterisk on two bottom blots likely represent defective interfering RNAs. 
$N b R d R P 1 m$-specific probe showed that the NbRdRPIm fragment was detected in the recombinant virus pTRdRP1mp19/wt in both inoculated leaves and upper, systemically infected leaves in two of three experiments (Fig. 3B). However, the overall accumulation of viral RNAs in both instances was relatively low.

\section{DISCUSSION}

Previous experiments using plant viruses as gene delivery vectors to silence transgenes or endogenous host genes led to the discovery that mRNAs of transgenes and host genes are degraded to an undetectable level if the nonviral fragment inserted in the genome of a plant virus shares the same sequence with the transgene or the host gene (Hiriart et al. 2003; Qiu et al. 2002; Ratcliff et al. 2001; Ruiz et al. 1998). These discoveries indicated that a foreign insert in a viral genome is an effective silencing inducer of a gene that is homologous to the foreign insert. On the other hand, to what extent foreign inserts in recombinant viruses are targeted by the gene-silencing pathway remains largely unknown. In this study, we investigated the integrity of foreign inserts that are targets of gene silencing in recombinant viruses by using a well-characterized TBSV and DI gene viral vector system (Hou and Qiu 2003; Qiu et al. 2002; Scholthof 1999). We observed that GFP-derived inserts were retained in the recombinant TBSV virus (pTG-p19/wt) only on GFP-transgenic $N$. benthamiana plants in which silencing of the GFP transgene was initiated. Interestingly, the same insert was removed from the virus on wild-type plants (Fig. 1). Similarly, the GFP-derived fragment was retained in the chimeric DI-P populations that were passed through GFPtransgenic $N$. benthamiana plants in which silencing of the GFP transgene occurred, whereas the same fragment was deleted from the DI-P populations that were passed through wild-type plants. Moreover, this process was independent of the presence of TBSV P19 (Fig. 2). It also was found that the foreign insert derived from the endogenous NbRdRPIm gene of $N$. benthamiana was retained in the recombinant TBSV virus, whereas the fragment of the $\mathrm{NbChlH}$ gene was deleted (Fig. 3B), implying that the removal of foreign inserts from the TBSV recombinant virus is host gene specific.

A number of studies demonstrated that genomic recombination occurs frequently during TBSV infections, presumably because the replication complex of TBSV tends to switch templates during the replication process (Cheng and Nagy 2003; White and Morris 1999), especially in experimental N. benthamiana plants. The propensity of TBSV to recombine leads to abundant accumulation of DIs that commonly are detected in TBSV-infected $N$. benthamiana plants (Hillman et al. 1987; White and Morris 1999). The tendency of the TBSV replication complex to recombine different regions also can result in the formation of TBSV mutants (Desvoyes and Scholthof 2002). Recombination of the genomic RNA of a TBSV derivative containing a defective $\mathrm{CP}$ gene with a transgenically expressed CP mRNA also produced wild-type TBSV in these CP-transgenic N. benthamiana plants (Borja et al. 1999). Closely related to frequent recombination events is the phenomenon that TBSV recombinant viruses rapidly remove the entire fragment or major portion of nonviral sequences (Qiu et al. 2002; Scholthof 1999; Scholthof et al. 1993). This feature could reflect a selective advantage of TBSV by avoiding the amplification of nonessential elements (Pogue et al. 2002; Scholthof 1999). Consequently, it is not surprising to observe that upper, noninoculated leaves of wild-type $N$. benthamiana plants became infected with TBSV viruses devoid of inserted nonviral fragments that were derived from GFP or sGFP genes (Fig. 1B) as well as from an endogenous $\mathrm{NbChlH}$ gene (Fig.
3B). Furthermore, the recombination process of TBSV to remove foreign inserts also occurs in the subviral DI-P population from which the inserted GFP fragment was removed after the DI-P population was passed through wild-type $N$. benthamiana plants three times (Fig. 2).

Intriguingly, the GFP gene-derived fragment was retained in the chimeric pTG-p19/wt virus or DI-P only on the GFP-transgenic $N$. benthamiana plants in which silencing of the GFP transgene was simultaneously initiated. As discussed earlier, wild-type TBSV viruses frequently are regenerated in N. benthamiana plants that were transformed with the functional TBSV CP gene (Borja et al. 1999). This occurs possibly via a double recombination event between the CP transgene mRNA and the TBSV mutant in which a small fragment of the $\mathrm{CP}$ gene was deleted (Borja et al. 1999). Therefore, one possibility is that the maintenance of the GFP fragment in the chimeric pTG-p19/wt virus or DI-P in the GFP-transgenic plants may be a result of ongoing recombination events that incorporate a fragment of GFP mRNA back into the pTG-p19/wt virus or DI-P. Although this possibility may exist, one also could argue against it. The TBSV CP has been shown to play an auxiliary role in the systemic movement of the virus (Desvoyes and Scholthof 2002; Qu and Morris 2002; Scholthof et al. 1993). Therefore, in the TBSV CP-transgenic plants, the restored wild-type TBSV has a selective advantage over the TBSV mutant that is incapable of expressing the functional TBSV CP. On the other hand, recombination between the GFP mRNA and the TBSV mutant pTG-p19/wt has no selective advantage for the progeny viruses. Instead, the recombinants are vulnerable to the gene-silencing machinery, as in the case of PVXGFP gene vector, whereby the chimeric RNA genome was eliminated by the silencing machinery (Ruiz et al. 1998; Thomas et al. 2001). Presumably, this occurred because the recombinant virus contained the very sequence that is subjected to the degradation once silencing of the target GFP gene is initiated. The same argument also can be applied to DI-P that contains the target GFP sequence of gene silencing. A recent study showed that cis elements dispensable for replication frequently are removed during formation of DIs, and it was proposed that VIGS provides selection pressure against DIs containing VIGS target sequences (Szittya et al. 2002). Therefore, it could be expected that DI-Ps should be outcompeted by the de novogenerated DIs devoid of the GFP fragment that accumulated abundantly in the mixed virus population, but this does not occur in GFP transgenic plants (Fig. 2B). In addition, gradual degradation of GFP mRNA as a result of silencing of the GFP transgene may prevent the recombination from frequently occurring between GFP mRNAs and recombinant TBSV or DIs .

Within this context, we propose that, as an innate property of multiplying true-to-original viral variants, the TBSV replication complex tends to eliminate nonviral sequences. This property, however, is compromised under conditions when the inserted nonviral fragments are the very targets of gene silencing via mechanisms that are yet to be explored. In other words, silencing of the inserted nonviral fragments hinders the recombination events of TBSV that otherwise could remove nonviral RNAs in the tissues of wild-type plants in which silencing of the target gene does not exist. By extending the observation that the recombination event is host dependent (Desvoyes and Scholthof 2002; Omarov et al. 2004), our results support the proposition that TBSV recombination also is influenced by silencing of the target genes.

Considering that there is also a selective advantage for a smaller virus genome that moves more efficiently (Pogue et al. 2002), it cannot be ruled out that the route for systemic movement of chimeric TBSV viruses also may play a role in selective transfer of TBSV viruses. It is likely that vascular tissues 
preferentially allow TBSV variants that are devoid of nonviral fragments to pass, but this restriction is relaxed in vascular tissues undergoing silencing of nonviral fragments. Regardless of the possible mechanisms, this study reveals a phenomenon that nonviral inserts are retained in TBSV and DIs population in plants undergoing silencing of the very foreign inserts, and the integrity of nonviral fragments in the recombinant viruses also is dependent on the type of inserted host gene.

\section{MATERIALS AND METHODS}

\section{Plant material.}

GFP-transgenic $N$. benthamiana line $16 \mathrm{C}$ was obtained from D. C. Baulcombe (Sainsbury Laboratory, Norwich, U.K.). The transgenic $N$. benthamiana line $16 \mathrm{C}$ carries one copy of the 35S:GFP transgene at a single locus that is homozygous (Ruiz et al. 1998). GFP-transgenic and wild-type $N$. benthamiana plants were grown under conditions of $14 \mathrm{~h}$ of light at $24^{\circ} \mathrm{C}, 10$ $\mathrm{h}$ of dark at $20^{\circ} \mathrm{C}$, and light intensity of $126 \mu \mathrm{E} \mathrm{m}^{-2} \mathrm{~s}^{-1}$.

\section{Chimeric TBSV and DI-P constructs.}

An infectious full-length cDNA clone, pTBSV-100, of the TBSV cherry strain was used as the prototype TBSV (Hearne et al. 1990). The plasmid pHS157 is a TBSV-derived mutant in which the expression of functional P19 is blocked by the introduction of a stop codon at amino acid position 52 (Scholthof et al. 1995c). The detailed description of constructs pTG-p19/wt and DI-P can be found in a previous study (Qiu et al. 2002). The construct pTsG-p19/wt was modified from a previously constructed TBSV-sGFP that is incapable of expressing P19 (Sit et al. 1998) by replacing the nonfunctional p19 gene with the original TBSV p19 gene.

The pTb549s plasmid that contains a 549-bp fragment of the $\mathrm{NbChlH}$ gene encoding the $\mathrm{H}$ subunit of magnesium chelatase of $N$. benthamiana plant was kindly provided by Kirsi Lehto (University of Turku, Finland). The primers pTB549s (5'cgtcgactctccttgaccgagcagtc, SalI site was underlined) and pTB549as (5'-ggagctcgccttcataccacttggggt) were used to amplify the 549-bp fragment of the $\mathrm{NbChlH}$ gene (hereinafter referred to as ChlH-549) from pTb549s plasmids. The ChlH-549 fragment then was cloned into the pGEM-T-Easy vector (Promega Corp., Madison, WI, U.S.A.). Subsequently, the ChlH-549 fragment was released from the recombinant pGEM-T-Easy plasmid with digestion of restriction enzymes SalI and PstI, and inserted into the TBSV gene vector pTBSVsGFP/p19 that was cut with $\mathrm{XhoI}$ and $P$ stI restriction enzymes. This cloning process resulted in the pTChlH-p19/wt construct.

A 776-bp fragment of an RNA-dependent RNA polymerase gene (NbRdRPIm) was isolated from $N$. benthamiana plants and inserted into the pCR4-TOPO vector (Invitrogen, Carlsbad, CA, U.S.A.) to create plasmid pB22. To generate the pTRdRP1m-p19/wt construct, the 776-bp fragment of NbRdRP1m was amplified from the plasmid pB22 DNA with two primers NbRdRPs (5'-ggtcgacaagtgattggatgggatatc, SalI site is underlined) and NbRdRPas (5'-gatgatatgtaacatggtaccaag $\mathrm{ccg}$ ). The resultant 776-bp fragment of $N b R d R P 1 m$ was inserted between the XhoI and PstI sites of the TBSV gene vector pTBSV-sGFP/p19 to form pTRdRP1m-p19/wt.

\section{Transcript inoculation and passages of virus populations.}

In vitro transcription reactions to produce uncapped transcripts of TBSV, pHS157, pTG-p19/wt, pTsG-p19/wt, pTChlH-p19/wt, pTRdRP1m-p19/wt, and DI-P constructs and the inoculation of transcripts onto $N$. benthamiana plants were performed as described previously (Scholthof et al. 1995a and c). Three plants were inoculated with transcripts in each experiment. Two leaves of each plant were inoculated.
When local and systemic symptoms appeared on plants that were inoculated with transcripts, sap inoculum for subsequent transfer was prepared by grinding $0.1 \mathrm{~g}$ of symptomatic inoculated or systemically infected leaves in $0.5 \mathrm{ml}$ of virus inoculation buffer (0.05 M sodium phosphate, $\mathrm{pH} 7.0,1 \%$ Celite).

\section{RNA gel blot analyses.}

Total RNA was extracted from both inoculated leaves and upper, systemically infected leaves. For plants inoculated with a mixture of DI-P transcripts and TBSV or pHS157 transcripts, total RNA was extracted at 7 dpi. For plants inoculated with pTG-p19/wt, pTsG-p19/wt, pTChlH-p19/wt, and pTRdRP1mp19/wt transcripts, total RNA was extracted from inoculated leaves at $7 \mathrm{dpi}$ and from systemically infected leaves at $14 \mathrm{dpi}$. RNA blot assays were performed as described previously (Qiu et al. 2002). GFP-, sGFP-, NbChlH-, NbRdRP-, and TBSVspecific probes were synthesized by the random-primed labeling method and then used to hybridize the nylon membranes that were blotted with RNAs.

\section{ACKNOWLEDGMENTS}

We are grateful to H. Scholthof (Texas A\&M University, College Station) for providing TBSV and DI-P constructs, D. Baulcombe (Sainsbury Laboratory, Norwich, U.K.) for providing seed of GFP gene-transgenic $N$. benthamiana, T. Sit for the pTsG plasmid, and K. Lehto (University of Turku, Finland) for the pTb549s plasmid. We are grateful to H. Scholthof and F. Qu for their helpful suggestion on the experiments. We thank L. Kovacs and M. Odneal for critically reading the manuscript. This work is supported by the Research Foundation and the Graduate College of the Southwest Missouri State University.

\section{LITERATURE CITED}

Baulcombe, D. C. 1999. Fast forward genetics based on virus-induced gene silencing. Curr. Opin. Plant Biol. 2:109-113.

Borja, M., Rubio, T., Scholthof, H. B., and Jackson, A. O. 1999. Restoration of wild-type virus by double recombination of Tombusvirus mutants with a host transgene. Mol. Plant-Microbe Interact. 12:153-162.

Cheng, C. P., and Nagy, P. D. 2003. Mechanism of RNA recombination in carmo- and tombusviruses: Evidence for template switching by the RNA-dependent RNA polymerase in vitro. J. Virol. 77:12033-12047.

Chiu, W.-L., Yasuo, Y., Zeng, W., Hirano, T., Kobayashi, H., and Sheen, J. 1996. Engineered GFP as a vital reporter in plants. Curr. Biol. 6:325-330.

Desvoyes, B., and Scholthof, H. B. 2002. Host-dependent recombination of a Tomato bushy stunt virus coat protein mutant yields truncated capsid subunits that form virus-like complexes which benefit systemic spread. Virology 304:434-442.

Faivre-Rampant, O., Gilroy, E. M., Hrubikova, K., Hein, I., Millam, S., Loake, G. J., Birch, P., Taylor, M., and Lacomme, C. 2004. Potato virus $\mathrm{X}$-induced gene silencing in leaves and tubers of potato. Plant Physiol. 134:1308-1316.

Hearne, P. Q., Knorr, D. A., Hillman, B. I., and Morris, T. J. 1990. The complete genome structure and synthesis of infectious RNA from clones of tomato bushy stunt virus. Virology 177:141-151.

Hillman, B. I., Carrington, J. C., and Morris, T. J. 1987. A defective interfering RNA that contains a mosaic of a plant virus genome. Cell 51:427-434.

Hiriart, J.-B., Aro, E.-M., and Lehto, K. 2003. Dynamics of the VIGS-mediated chimeric silencing of the Nicotiana benthamiana ChlH gene and of the tobacco mosaic virus vector. Mol. Plant-Microbe Interact. 16:99-106.

Hiriart, J.-B., Lehto, K., Tyystjarve, E., Junttila, T., and Aro, E.-M. 2002. Suppression of a key gene involved in chlorophyll biosynthesis by means of virus-induced gene silencing. Plant Mol. Biol. 50:213-224.

Hou, H. S., and Qiu, W. P. 2003. A novel co-delivery system consisting of a Tomato bushy stunt virus and a defective interfering RNA for studying gene silencing. J. Virol. Methods 111:37-42.

Liu, Y., Schiff, M., and Dinesh-Kumar, S. P. 2002. Virus-induced gene silencing in tomato. Plant J. 31:777-786.

Omarov, R. T., Rezende, J. A. M., and Scholthof, H. B. 2004. Host-specific generation and maintenance of Tomato bushy stunt virus defective interfering RNAs. Mol. Plant-Microbe Interact. 17:195-201.

Pogue, G. P., Lindbo, J. A., Garger, S. J., and Fitzmaurice, W. P. 2002. Making an ally from an enemy: Plant virology and the new agriculture. Annu. Rev. Phytopathol. 40:45-74. 
Qiu, W. P., Park, J.-W., and Scholthof, H. B. 2002. Tombusvirus P19-mediated suppression of virus induced gene silencing is controlled by genetic and dosage features that influence pathogenicity. Mol. PlantMicrobe Interact. 15:269-280.

Qu, F., and Morris, T. J. 2002. Efficient infection of Nicotiana benthamiana by Tomato bushy stunt virus is facilitated by the coat protein and maintained by p19 through suppression of gene silencing. Mol. PlantMicrobe Interact. 15:193-202.

Rabindran, S., and Dawson, W. O. 2001. Assessment of recombinants that arise from the use of a TMV-based transient expression vector. Virology 284:182-189.

Ratcliff, F., Harrison, B. D., and Baulcombe, D. C. 1997. A similarity between viral defense and gene silencing in plants. Science 276:1558-1560.

Ratcliff, F., Martin-Hernandez, A. M., and Baulcombe, D. C. 2001. Tobacco rattle virus as a vector for analysis of gene function by silencing. Plant J. 25:237-245.

Ruiz, M. T., Voinnet, O., and Baulcombe, D. C. 1998. Initiation and maintenance of virus-induced gene silencing. Plant Cell 10:937-946.

Russo, M., Burgyan, J., and Martelli, G. P. 1994. Molecular biology of Tombusviridae. Adv. Virus Res. 44:381-428.

Scholthof, H. B. 1999. Rapid delivery of foreign genes into plants by direct rub-inoculation with intact plasmid DNA of a tomato bushy stunt virus gene vector. J. Virol. 73:7823-7829.

Scholthof, H. B., Morris, T. J., and Jackson, A. O. 1993. The capsid protein gene of tomato bushy stunt virus is dispensable for systemic movement and can be replaced for localized expression of foreign genes. Mol. Plant-Microbe Interact. 6:309-322.

Scholthof, H. B., Scholthof, K.-B. G., and Jackson, A. O. 1995a. Identification of tomato bushy stunt virus host-specific symptom determinants by expression of individual genes from a potato virus $\mathrm{X}$ vector. Plant Cell 7:1157-1172.

Scholthof, K.-B., G., Scholthof, H. B., and Jackson, A. O. 1995b. The tomato bushy stunt virus replicase proteins are coordinately expressed and membrane associated. Virology 208:365-369.

Scholthof, H. B., Scholthof, K.-B., G., Kikkert, M., and Jackson, A. O 1995c. Tomato bushy stunt virus spread is regulated by two nested genes that function in cell-to-cell movement and host-dependent systemic invasion. Virology 213:425-438.

Scholthof, H. B., Scholthof, K.-B. G., and Jackson, A. O. 1996. Plant virus gene vectors for transient expression of foreign proteins in plants. Annu. Rev. Phytopathol. 34:299-323.

Silhavy, D., Molnar, A., Lucioli, A., Szittya, G., Hornyik, C., Tavazza, M. and Burgyan, J. 2002. A viral protein suppresses RNA silencing and binds silencing-generated, 21- to 25-nucleotide double-stranded RNAs. EMBO (Eur. Mol. Biol. Organ.) J. 21:3070-3080.

Sit, T. L., Vaewhongs, A. A., and Lommel, S. A. 1998. RNA-mediated trans-activation of transcription from a viral RNA. Science 281:829832.

Szittya, G., Molnar, A., Silhavy, D., Hornyik, C., and Burgyan, J. 2002. Short defective interfering RNAs of Tombusviruses are not targeted but trigger post-transcriptional gene silencing against their helper virus. Plant Cell 14:359-372.

Thomas, C. L., Jones, L., Baulcombe, D. C., and Maule, A. J. 2001. Size constraints for targeting post-transcriptional gene silencing and for RNA-directed methylation in Nicotiana benthamiana using a potato virus X vector. Plant J. 25:417-425.

Voinnet, O., Pinto, Y. M., and Baulcombe, D. C. 1999. Suppression of gene silencing: A general strategy used by diverse DNA and RNA viruses of plants. Proc. Natl. Acad. Sci. U.S.A. 96:14147-14152.

Walker, C. J., and Willows, R. D. 1997. Mechanism and regulation of Mgchelatase. Biochem. J. 327:321-333.

Waterhouse, P. M., Wang, M.-B., and Lough, T. 2001. Gene silencing as an adaptive defense against viruses. Nature 411:834-842.

White, K. A., and Morris, T. J. 1999. Defective and defective interfering RNAs of monopartite plus-strand RNA plant viruses. Pages 1-17 in: Current Topics in Microbiology and Immunology: Satellites and Defective RNAs. P. K. Vogt and A. O. Jackson, eds. Springer, New York.

Yang, S.-J., Carter, S. A., Cole, A. B., Cheng, N.-H., and Nelson, R. S. 2004. A natural variant of a host RNA-dependent RNA polymerase is associated with increased susceptibility to viruses by Nicotiana benthamiana. Proc. Natl. Acad. Sci. U.S.A. 101:6297-6302.

\section{Erratum}

A correction was made to this article on August 10, 2005. Figures 1 and 2 were replaced with images containing grayscale. 